\title{
CONCEPTS
}

\section{Orbital Compartment Syndrome: Alternative Tools to Perform a Lateral Canthotomy and Cantholysis}

\author{
Kenneth V. Iserson, MD, MBA; Zelda Luke-Blyden, MD; Scott Clemans, BSME \\ From the Department of Emergency Medicine, University of Arizona, Tucson, AZ and the GPHC Emergency Medicine Residency, Georgetown, \\ Guyana (Dr Iserson); Department of Emergency Medicine, Georgetown Public Hospital Corporation, Georgetown, Guyana (Dr Luke-Blyden); \\ and Buecher Biological Consulting, Tucson, AZ (Mr Clemans).
}

\begin{abstract}
Orbital compartment syndrome acutely threatens vision. Lateral canthotomy and cantholysis ameliorate the compartment syndrome and, to save a patient's vision, must be performed in a timely manner. This requires appropriate tools. In resource-poor settings, the straight hemostat and iris scissors that are generally used for this procedure may be unavailable. In such situations, safe alternatives include using a multitool in place of a hemostat and a \#11 scalpel blade instead of the iris scissors. As when using hemostats of varying sizes, the pressure applied to the multitool must be carefully modulated. When using a scalpel blade for the lateral canthotomy, the hemostat arm remains beneath the lateral canthus as a "backstop" to protect deeper tissues. For the cantholysis, use the back of the blade to "strum" for the ligaments, reversing its direction only to cut the ligament when it is identified.
\end{abstract}

Key words: lateral canthotomy, cantholysis, orbital compartment syndrome, retrobulbar hematoma, ophthalmic trauma

\section{Introduction}

Orbital compartment syndrome acutely threatens vision by compressing the ophthalmic artery. Although there are numerous possible causes (Table), ${ }^{1,2}$ trauma-induced hemorrhage is the most common in patients presenting for emergency medical care. Since the early 1990s, lateral canthotomy and cantholysis has been the accepted method for emergency treatment of this condition. ${ }^{1}$ Although a standard, simple technique for this procedure exists using common equipment, these basic tools may be unavailable in resource-poor situations, such as remote areas or developing countries. Therefore, a method using alternative equipment is necessary. In this case, emergency physicians in a resource-poor, very busy emergency department (ED) quickly devised a method to safely perform a lateral canthotomy and cantholysis using tools often available in austere settings. This improvisation offers a viable alternative method for those needing to perform this procedure.

\section{Lateral Canthotomy/Cantholysis: Case}

A 46-year-old man with a blunt cranial and globe injury arrived in the ED unconscious (Glasgow Coma Score $=5$ )

Corresponding author: Kenneth V. Iserson, MD, MBA, Department of Emergency Medicine, University of Arizona, 4930 N. Calle Faja, Tucson, AZ 85718 (e-mail: kvi@u.arizona.edu). and with obvious proptosis. As head injury treatment was initiated, the eyes were evaluated, showing that they were both midpoint and nonreactive to light, with the left eye proptotic and resistant to retropulsion with light digital orbital compression. There was no tonometer. It was uncertain whether the eye was salvageable, but the globe appeared intact. No ophthalmologist was immediately available.

A lateral canthotomy and cantholysis was indicated. However, in our resource-poor setting, there were no iris or similar scissors, a key part of the standard procedure. Even so, the clinicians successfully performed the procedure using a \#11 scalpel blade. No anesthesia was needed as the patient was unconscious. Although no vasoconstrictor was used, only minimal bleeding ensued when cutting the lateral canthus, and it was easily controlled with minimal pressure over the bony orbit. As is usual, minimal blood emerged from the orbit.

The hospital ophthalmologist subsequently assessed the procedure as having been "done perfectly." The patient died of his head injuries several days later without regaining consciousness.

\section{Discussion}

\section{RELEVANT ORBITAL ANATOMY}

The orbital cavity is a $30-\mathrm{mL}, 4$-sided pyramid enclosing the globe, with the superior and inferior orbital septa 
Table. Causes of orbital compartment syndrome ${ }^{1,2}$

Traumatic retrobulbar bleeding
Acute orbital/facial trauma (most common)
Eyelid surgery
Intranasal or external ethmoidectomy
Recent retrobulbar anesthesia
Spontaneous retrobulbar bleeding/hematomas
Atherosclerosis
Hemophilia
Hypertension
Intraorbital aneurysm of the ophthalmic artery
Leukemia
Valsalva maneuvers
Venous anomalies
Von Willebrand's disease
Less common conditions
Orbital abscess
Orbital cellulitis
Orbital emphysema
Orbital inflammation
Tumors

anteriorly (Figure 1). The walls are composed of 7 bones (maxilla, palatine, zygomatic, sphenoid, frontal, ethmoid, and lacrimal). The globe itself has a volume of $7 \mathrm{~mL}$, with the optic nerve and ophthalmic artery tethering it posteriorly, limiting forward movement. ${ }^{3}$

The medial and lateral canthal tendons attach the muscular layer of the eyelids to the bone at the orbital rim (Figure 2). ${ }^{4}$ They further prevent any anterior globe displacement. ${ }^{5}$ These tendons often are termed the medial and lateral canthal (or palpebral) ligaments, although they are actually muscular insertions into the bone. ${ }^{4}$ Superficial to the lateral canthal tendon and barely separated from it is a fibrous fascial plane, the lateral canthal raphe. The inferior and superior canthal

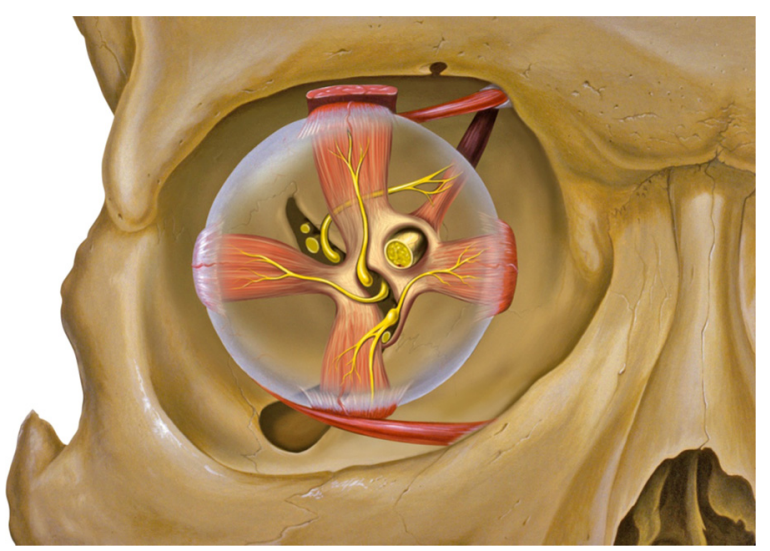

Figure 1. Orbit, globe, and adjacent structures. (Peter J. Lynch, Medical Illustrator, Creative Commons. Uploaded Dec. 23, 2006). ligaments run from the orbital periosteum to tissues of the interior lids.

\section{ORBITAL COMPARTMENT SYNDROME}

Increased pressure within the orbital cavity is an emergency that requires prompt diagnosis and intervention to prevent blindness. Retrobulbar bleeding is the most common cause of orbital compartment syndrome, causing blindness in nearly half of patients who lose their vision after orbital or facial trauma. ${ }^{6}$ Bleeding within this cavity compromises neurovascular structures passing into the orbit because there is little room for decompression. Although the optic nerve is very sensitive to reduced orbital perfusion pressure, the retina may be at greatest risk from central retinal artery occlusion. ${ }^{3}$ Without decompression, which is "the facial equivalent of a fasciotomy in an injured extremity,", irreversible vision loss may occur in as little as 90 to 120 minutes. 8

By 1976, ophthalmologists were aware that a canthotomy and cantholysis could relieve lid pressure on the globe. ${ }^{4}$ In 1994, Yung et al. ${ }^{9}$ demonstrated that lateral canthotomy combined with inferior cantholysis provides a significantly greater decrease in intraocular pressure (IOP) than either procedure alone. Because these techniques are uncommon in EDs, practitioners have learned and practiced them on sheep and primate models and on human cadavers.

\section{IDENTIFYING PATIENTS WITH ORBITAL COMPARTMENT SYNDROME}

Emergency physicians must recognize orbital compartment syndrome and treat it in a timely manner to avoid or reverse visual loss. ${ }^{8}$ A history of orbital or facial trauma and symptoms such as eye pain, decreased visual acuity, diplopia, or lack of motility (in conscious patients) strongly suggest the diagnosis. Digital orbital compression, although highly inaccurate, may indicate a resistance to retropulsion. Although infraorbital hypesthesia is frequently cited as a key symptom of orbital compartment syndrome, it may be difficult to assess and its clinical significance is minimal because it is found in many conditions. If an orbital compartment syndrome diagnosis is probable, especially with decreased visual acuity, clinicians should not delay the release procedures to obtain imaging because irreversible vision loss may occur if retina ischemia time is greater than 90 to 120 minutes.

As in the case presented, many such patients have concomitant head injuries, so clinicians may need to suspect this diagnosis based only on physical findings. These may include limited extraocular movements 


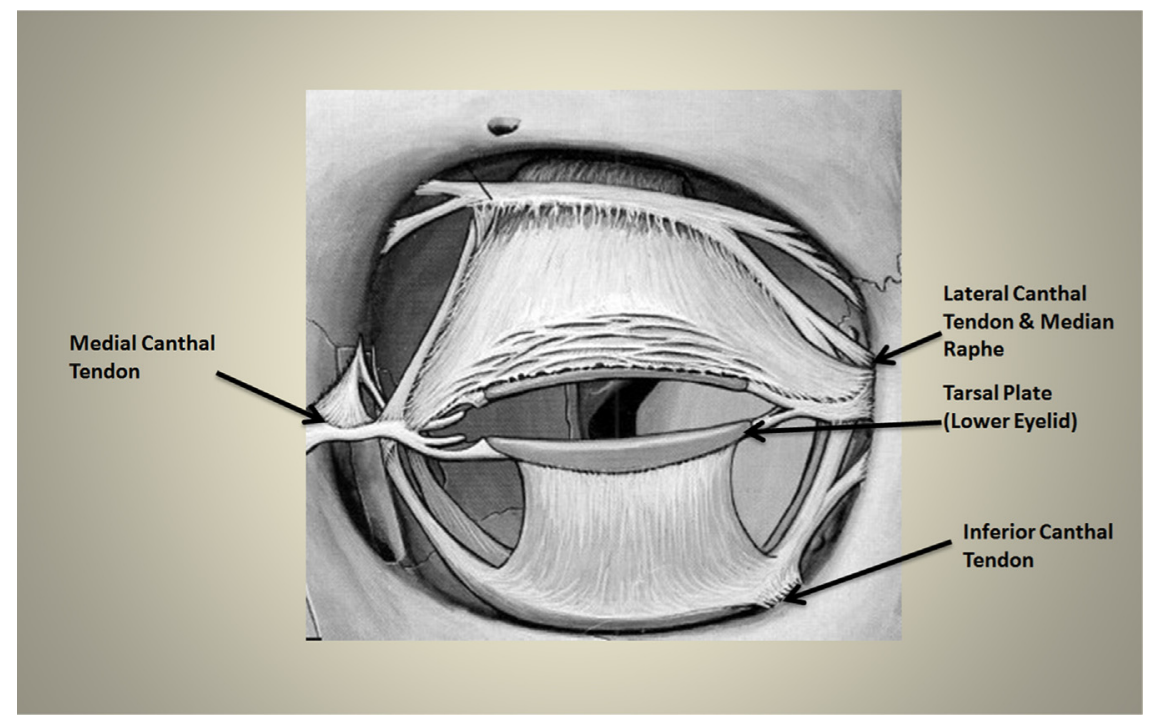

Figure 2. Soft tissue structures around the anterior globe.

(ophthalmoplegia), proptosis, ecchymosis around the eye, bloody chemosis, increased intraocular pressure (IOP > $40 \mathrm{~mm} \mathrm{Hg}$; normal IOP is $10-21 \mathrm{~mm} \mathrm{Hg}$ ), resistance to retropulsion (pushing the globe deeper into the orbit through the closed eyelids), diffuse subconjunctival hemorrhage, or an afferent pupillary defect (test the afferent papillary defect with the swinging flashlight test, which is positive when both the normal and abnormal pupils dilate in response to light in the affected eye). Although a head injury might confound interpretation of the pupillary examination, it still provides valuable information. Pupillary responses that are normal only in the uninjured eye indicate that the abnormal papillary response is caused by an eye injury rather than brain injury. In addition, even if the patient never regains consciousness, restoration of the papillary response indicates a successful canthotomy/cantholysis. Less definitive findings include marked periorbital edema or crepitus, a cherry-red macula, and optic nerve head pallor. $^{10,11}$

Frequently cited in the literature, but without evidence, is the suspicion that a globe rupture may be a contraindication for these procedures. ${ }^{1}$ However, because globe ruptures usually cause low IOP and enophthalmos without proptosis, it is unlikely that a retrobulbar hemorrhage would be emergently identified in these cases.

\section{LATERAL CANTHOTOMY AND CANTHOLYSIS IN STANDARD SETTINGS}

Although rarely done in emergency settings, lateral canthotomy and cantholysis are easier to perform than many common ED procedures because every patient's lateral canthal and canthal tendons are in identical anatomic positions. While gathering materials for the procedure, elevate the patient's head to decrease IOP and intracranial pressure. If the procedure can be performed with the patient in this position, that is optimal. Patients who cannot fully cooperate must be sedated.

If time permits, clean and drape the lateral canthal area, although simple irrigation with normal saline solution is sometimes recommended in emergency cases. ${ }^{1}$ Irrigate the eye with normal saline solution to obtain a clean procedural field. If the patient is conscious and the surrounding tissues are not already numb from the orbital pressure, inject $1 \mathrm{~mL}$ of lidocaine (with 1\%-2\% epinephrine, if available, for hemostasis) or an alternative anesthetic ${ }^{12}$ into the lateral canthus. Direct the needle tip toward the lateral orbital rim; begin injecting when the needle touches bone (Figure 3A).

In standard settings, the next step is to reduce bleeding by placing a straight clamp (eg, Kelly hemostat) horizontally at the lateral corner of the patient's eye, with 1 blade under the orbital skin. (Figure 5 demonstrates this using a multitool, as described below.) Use this to clamp the 1 to $2 \mathrm{~cm}$ of skin from the corner of the eye to the bony orbit for 1 to 2 minutes. This provides hemostasis and marks the incision line for the lateral canthotomy. Then use a pair of iris or similar scissors (small blunttipped scissors may be safest) to completely cut through this tissue (Figure 3B).

To relieve lid pressure on the underlying globe, first divide the conjoined lateral canthal tendon band. This 


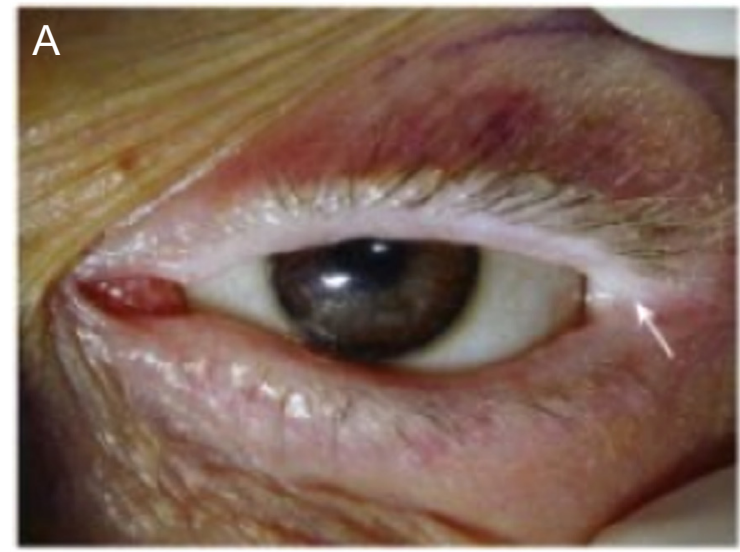

Arrow: Site for anesthetic \& crushing lateral orbital tissue.

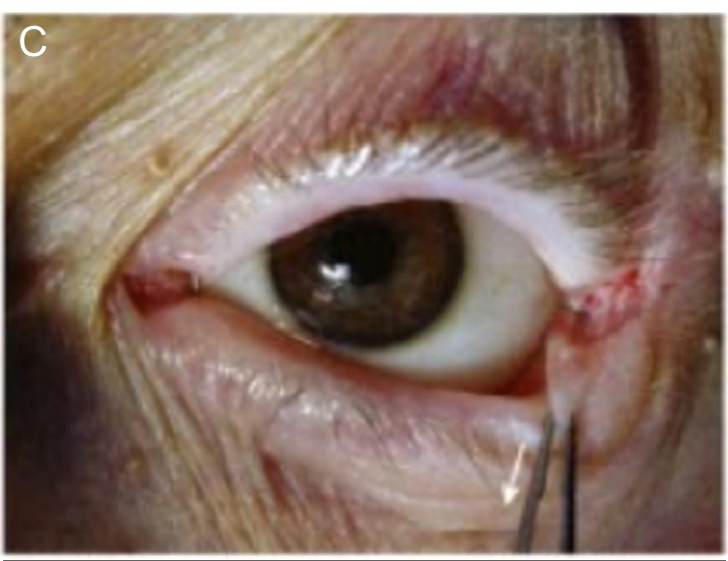

Pull the lower eyelid down, strum \& cut inferior tendons.

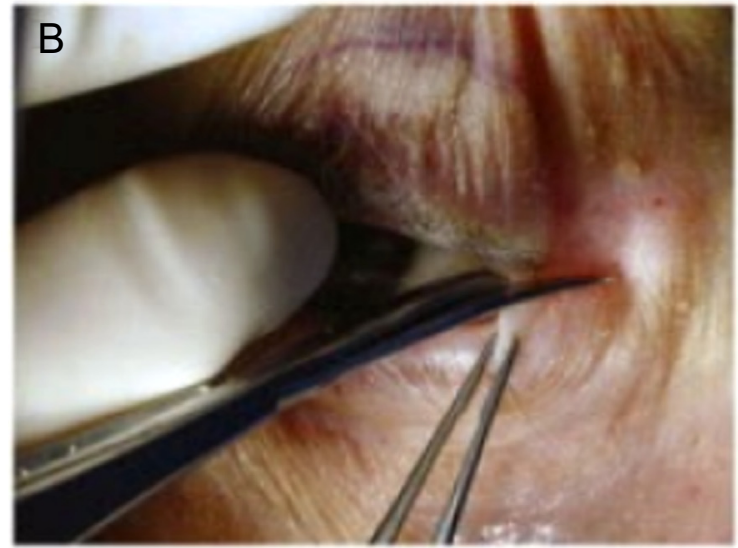

Cut lateral canthal tendon with scissors or knife.

Figure 3. Steps in a lateral canthotomy and cantholysis. A, Arrow: Site for anesthetic and crushing lateral orbital tissue. B, Cut lateral canthal tendon with scissors or knife. C, Pull the lower eyelid down, strum, and cut inferior tendons. Permission for photo montage (captions changed): Sarwark J. Procedures in Lateral Canthotomy. www.emcurious.com/blog-1/2014/9/25/the-lateral-canthotomy. Accessed August 29 , 2015.

procedure removes the lower lid's attachment to the lateral orbital wall, making it easy to pull away from the lid margin. ${ }^{1}$

The next step is to pull the inferior, and then often the superior, eyelid away from the globe and incise the inferior, and often the superior, canthal tendons (Figure 3C). Identify them by placing the iris scissors tips in the lateral one-third of the space between the globe and the retracted eyelid, carefully spreading them through the very lax and delicate subcutaneous areolar tissue. This process is sometimes described as "strumming" because encountering the tendons feels like plucking guitar strings, which are about the same width. ${ }^{7}$ It is much easier to feel than to visualize. Once the tendon is cut, consider doing the same procedure under the upper eyelid.
These procedures result in little morbidity and can later be repaired easily. ${ }^{7}$

\section{PROCEDURE IN RESOURCE-POOR SETTINGS}

As in this case, practitioners may need to perform a lateral canthotomy and cantholysis in resource-poor settings. In those situations, they may need to use unconventional local anesthetics and alternatives for a hemostat and scissors. Multiple local anesthetic substitutes have been described previously, and at least one is generally available in every setting where needed. ${ }^{12}$

Hemostats, the recommended tool to clamp the lateral orbital tissues, are not themselves uniform medical instruments. The force applied by the distal end of a hemostat or a similarly constructed device (eg, multitool, 
To substitute a multitool for a hemostat, it is necessary to calculate where on the multitool handles to apply the force so that it equals the force applied on the hemostat handles. (This assumes you want to maintain the same clamping force.)

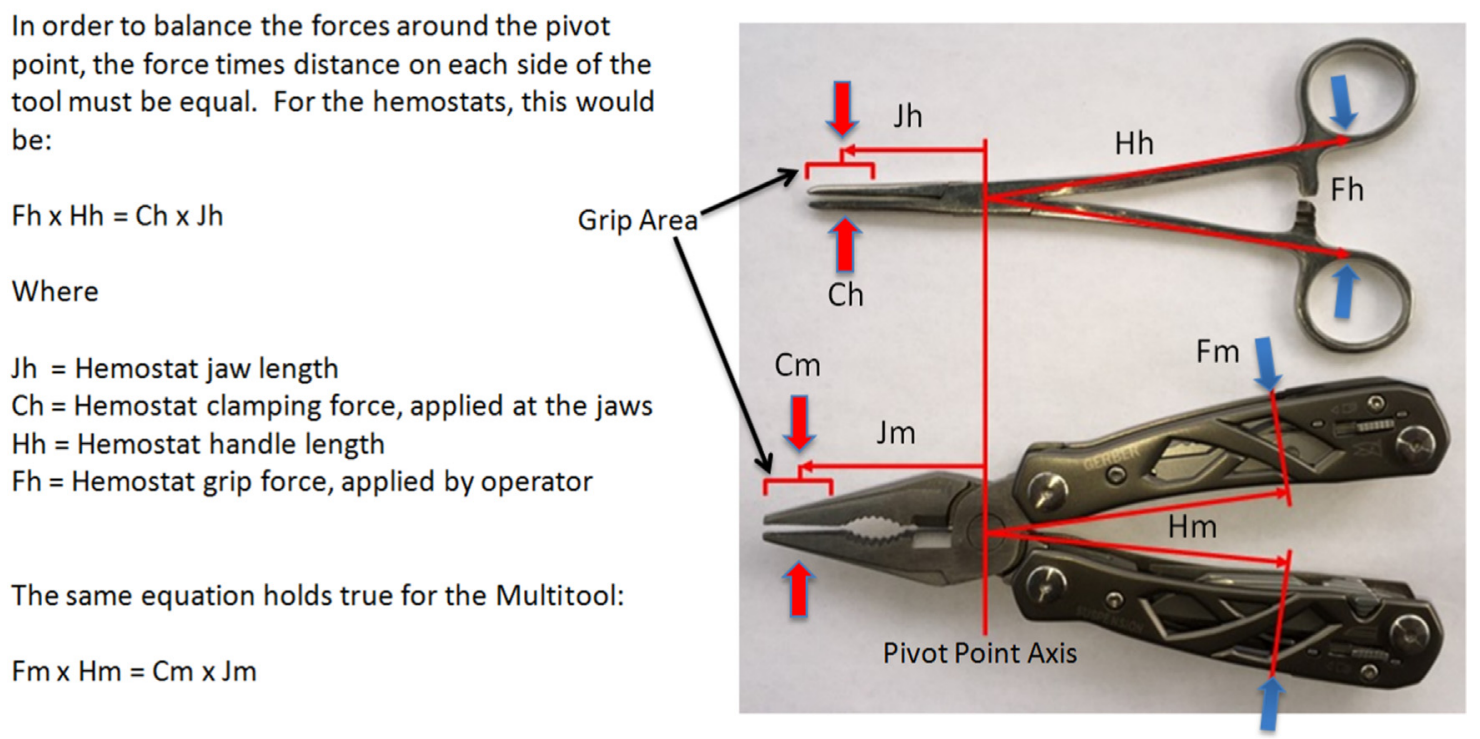

Figure 4. Description of force applied by hemostats and multitools. The clamping force varies with the placement of the operator's hand on the instrument and the pressure applied to the handle.

pliers) is directly related both to the operator-applied grip force and to the lengths of the handle and the end of the instrument from the pivot point as follows: clamping force on the tissues $=$ (operator-applied grip force $x$ handle length) / jaw length (Figure 4). This force also is directly proportional both to the distance from the operator's hand to the handle's pivot point and to how far the stepped bevel/locking mechanism on the hemostat is engaged.

This wide variation in tissue pressure suggests that using a clean multitool or similar nonmedical instrument to clamp the lateral orbital tissues may be suitable in resource-poor settings. A static model used to teach this procedure to wilderness medical providers used a multitool (eg, Leatherman, Gerber, SOG) to clamp simulated lateral canthal tissues fashioned from folded athletic tape. ${ }^{13}$ We have used the same tool to perform the procedure on an unembalmed cadaver with no apparent difference from using a hemostat that was clamped at the first stop.

In the case presented here, no small scissors were available to either incise the lateral tissues or identify and incise the inferior canthal tendon. As the alternative, we used a \#11 scalpel blade held with a short straight hemostat. We made this choice because of the blade's minimal width and its fine point, which permitted delicate movements in a small space (Figure 5). Its flat, noncutting back edge was suitable for identifying the canthal tissues. No emergency medicine literature mentions this, and in recent ophthalmic texts that discuss doing the procedures with a \#15 or \#11 blade, the authors cite neither personal experience nor literature to support this practice. ${ }^{7,14}$ Other similarly configured knife blades and small blunt-tipped scissors could also be used.

For the lateral canthotomy, the hemostat's interior arm was kept in place as a "backstop" to protect the eye when

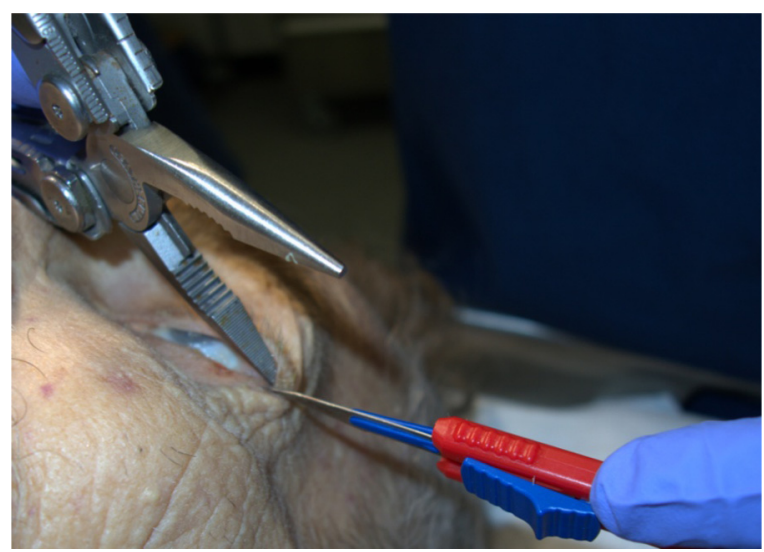

Figure 5. Lateral canthotomy with multitool and \#11 blade. 
incising the lateral canthal tissues with the scalpel blade. We could see that the tissues were completely incised when the hemostat moved through the space-something that is obvious when using scissors to cut the tissues.

As in this case, a cantholysis (cutting the inferior canthal tendon and then, if necessary, the superior tendon) usually follows the lateral canthotomy because the lateral canthotomy alone is often insufficient to decrease IOP. In this case, without a tonometer to measure whether the intraorbital pressure was greater than $40 \mathrm{~mm} \mathrm{Hg}$, we immediately proceeded to an inferior and then to a superior cantholysis.

Using the dull backside of the \#11 blade, we carefully pushed aside the alveolar tissues until we felt resistance from the tendon band (Figure 6). We then reversed the blade and cut the band. Because the intraorbital pressure was still thought to be significant, we repeated the process for the superior canthal ligaments (under the upper eyelid). Demonstrating that the procedures were successful, both eyelids were freely mobile after the procedures. ${ }^{10}$ If done with improvised instruments in the field, clinicians may consider using a systemic antibiotic until the patient is evacuated.

\section{COMPLICATIONS AND ALTERNATIVE TREATMENTS}

Complications of lateral canthotomy and cantholysis include iatrogenic globe injury from forceps or scissor tips; ptosis as a result of damage to the levator aponeurosis, which is located superiorly ${ }^{15}$; and injury to the lacrimal gland and lacrimal artery, which also lie superiorly. Less common complications include bleeding

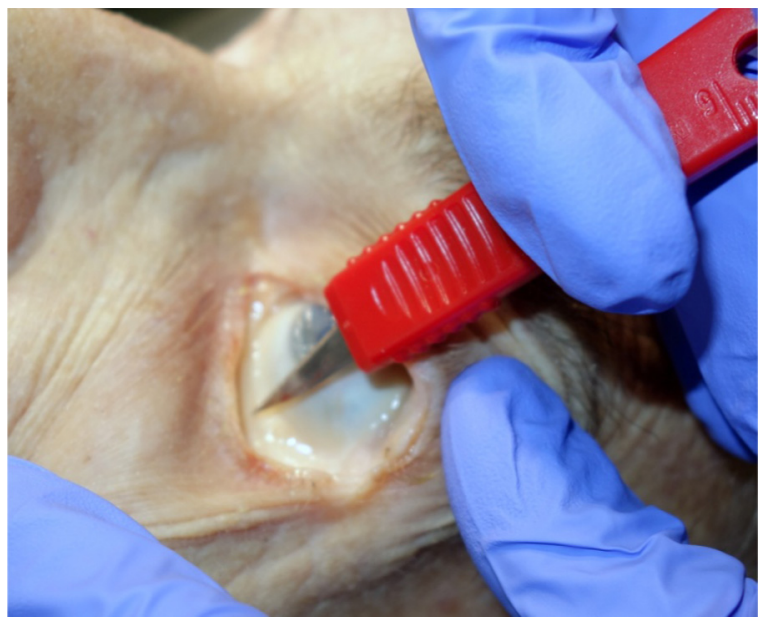

Figure 6. "Strumming" tendons with dull side of a \#11 blade. and infection. Extensive cantholysis may result in ectropion.

Although not very effective, medical therapy has been recommended for orbital compartment syndrome in cases when immediate surgical decompression was not possible or when increased IOP persists after surgical decompression. These measures include intravenous mannitol 20\% $2 \mathrm{~g} / \mathrm{kg}$ every 6 hours, acetazolamide 250 mg every 6 hours, or methylprednisolone $250 \mathrm{mg}$ every 6 hours.

Aside from treating associated trauma, general ED care for these patients includes providing local wound management, antibiotics, and tetanus immunization, as needed. Consult with an ophthalmologist as soon after the procedure as possible. If bleeding and intraocular hypertension continue, they may need to perform an anterior orbitotomy and drainage to evacuate hematoma (particularly, if subperiosteal) and to ligate or cauterize bleeding vessels. ${ }^{3}$ If the procedure has succeeded, the ophthalmologist will want to close the canthotomy when swelling has decreased, usually several days after the procedure. $^{1}$

\section{Acknowledgments}

Thanks go to two of my Tucson, AZ, colleagues, Leonard Joffe, MD, and Bob Buecher, Professional Engineer, for reviewing the manuscript.

\section{References}

1. Vassallo S, Hartstein M, Howard D, Stetz J. Traumatic retrobulbar hemorrhage: emergent decompression by lateral canthotomy and cantholysis. J Emerg Med. 2002;22: 251-256.

2. Rosdeutscher JD, Stadelmann WK. Diagnosis and treatment of retrobulbar hematoma resulting from blunt periorbital trauma. Ann Plast Surg. 1998;41:618-622.

3. McAllister AS. A clinical review of orbital anatomy and its relevance to retrobulbar anaesthesia. Cureus. 2013;5:e97.

4. Paton D, Goldberg MF. Management of Ocular Injuries. Philadelphia, PA: WB Saunders; 1976:19.

5. Lima V, Burt B, Leibovitch I, Prabhakaran V, Goldberg RA, Selva D. Orbital compartment syndrome: the ophthalmic surgical emergency. Surv Ophthalmol. 2009;54: 441-449.

6. Ansari MH. Blindness after facial fractures: a 19-year retrospective study. J Oral Maxillofac Surg. 2005;63: 229-237.

7. Vichweg TL. Face, Eye, and Ear Injuries. In: Martin MJ, Beekley AC, eds. Front Line Surgery: A Practical Approach. New York, NY: Springer; 2011:371-372. 
8. Popat H, Doyle PT, Davies SJ. Blindness following retrobulbar haemorrhage - it can be prevented. Br J Oral Maxillofac Surg. 2007;45:163-164.

9. Yung CW, Moorthy RS, Lindley D, Ringle M, Nunery WR. Efficacy of lateral canthotomy and cantholysis in orbital hemorrhage. Ophthal Plast Reconstr Surg. 1994;10: 137-141.

10. Winterton JV, Patel K, Mizen KD. Review of management options for a retrobulbar hemorrhage. J Oral Maxillofac Surg. 2007;65:296-299.

11. Straff D. Lateral Canthotomy. In: Shah K, Egan D, Quaas J, eds. Essential Emergency Trauma. Philadelphia, PA: Lippincott Williams \& Wilkins; 2010:419.
12. Iserson KV. Improvised Medicine: Providing Care in Extreme Environments New York, NY: McGraw-Hill; 2012;178-180.

13. Saxon KD, Kapadia AP, Juneja NS, Bassin BS. How to teach emergency procedural skills in an outdoor environment using low-fidelity simulation. Wilderness Environ Med. 2014;25:50-55.

14. Holmes S. Orbital Fractures. In: Perry M, Holmes S, eds. Atlas of Operative Maxillofacial Trauma Surgery. New York, NY: Springer; 2014:390.

15. Shek KC, Chung KL, Kam CW, Yau HH. Acute retrobulbar haemorrhage: an ophthalmic emergency. Emerg Med Australas. 2006;18:299-301. 